of the simple magnetic amplifier and showed a graphical method, analogous with that using the dynamic characteristic of the thermionic valve, for deducing the overall performance as a function of load and supply conditions. He then discussed feedback circuits and cascade operation and the effect of various factors on response time, pointing out that in the case of cascaded amplifiers this can be less than the sum of the individual response times. The author outlined the advantages of the magnetic amplifier in certain applications and concluded with descriptions of some typical cases.

Opening the next session, C. H. W. Brookes-Smith and J. A. Colls (Southern Instruments, Ltd.) read a paper on "Some Recent Improvements in Electronic Measuring Techniques" which dealt with the neasurement of pressure, stress, strain and displacement. They explained the frequency modulation system using a variable capacitance or induotance pick-up, which permits static calibration, is insensitive to random amplitude variations and which can have a level-response up to $30 \mathrm{kc} . / \mathrm{s}$. The difficulties oncountered in the design of a pick-up were discussed, and some types under development were described in detail. The authors pointed out that this system and the wire-resistance strain gauge cover most of the requirements of engineers and research workers. A compact drift-corrected D.C. amplifier was described which is intended for use with either system.

Dr. H. A. Dell (Mullard Electronic Instruments, Ltd.) closed the session with a paper on "The Measurement of some Transient Phenomena", transient being defined here as applying to events of short duration repeated at regular intervals. Two examples were given which illustrated some of the problems encountered in the design of equipment for the visual delineation on a cathode-ray oscillograph of such events having low and high recurrence-frequencies respectively.

The first example was a precision polarograph for chemical analysis work using a mercury dropping electrode. The voltage across the cell during the life of each drop is compared with a calibrated standard by means of a vibrating switch, and the result is presented on an oscilloscope with suitable afterglow characteristics. Dr. Dell's second example was that of a high-speed oscillograph for the examination of radar systems and the like, in which the period between successive events is long compared with the duration of a single event. Some circuits were described which fulfil the necessary time-relationship and which can present any selected portion of the wave-form under examination.

In the final session the first paper, entitled "An Industrial Servomechanism", by P. H. Briggs (Ferranti, Ltd.), gave details of an automatic control system for accurately guiding cloth being fed on to a pin stenter or drying machine; this could be applied to certain other processes, such as paper manufacture, involving a continuous web of material. Any lateral motion of the edge of the web is detected by a photocell and the necessary correction applied by a servo device, precautions being taken to ensure a quick response with freedom from hunting. The whole equipment is designed to operate under the conditions normally encountered in a textile mill and can be worked by relatively unskilled personnel, there being only one control knob to adjust. A commendable feature is that the electronic circuit, which is of simple design, can be used in other applice. tions.
Dr. A. J. Maddock (British Scientific Instrument Research Association) read the final paper, which was entitled "Co-operative Research at B.S.I.R.A.". He traced the history of the Association from the early days when it was concerned mainly with the problems of the optical industry, although investigations were made in other fields, two such being the production of phosphors for cathode-ray tube screens and a study of the magnetic susceptibility of the materials used for galvanometer suspensions. During the Second World War notable contributions were made to the production of graticules and to the antireflexion treatment of optical surfaces. The Association has recently expanded to seven departments in new premises at Chislehurst. Dr. Maddock described the facilities now available, with special reference to the Electricity and Electronics Departments, where work is in progress on such items as voltage stabilizers, D.c. and magnetic amplifiers, thermo-magnetic materials and on thermionic emission.

The session concluded with a summing-up by $\mathrm{Mr}$. A. J. Philpot, the director of the Scientific Instrument Manufacturers' Association, who said that the symposium had in every sense satisfied the dictionary definition of the word, and that it had now firmly established itself in the calendar with overy promise of increasing success in the future.

\section{RHEOLOGY OF EMULSIONS}

A JOINT meeting of the British Rheologists' Club and the Faraday Society was held at the Institute of Physies, 47 Belgrave Square, London, S.W.1, on November 11, Dr. G. W. Scott Blair being in the chair.

Dr. C. G. Sumner opened the discussion. He pointed out that the rheological behaviour of emulsions has much in common with that of suspensions, but certain distinctive features arise. By the use of suitable emulsifying agents, either of a given pair of immiscible liquids may be dispersed in the other, and the formation of droplets from bulk liquid is a hydrodynamic process into which the viscosities of both liquids enter. When an emulsion is caused to flow, deformation of the droplets may occur, which will reduce the apparent viscosity of the emulsion as a whole. It is doubtful whether any of the usual emulsifying machines breaks up only the liquid to be dispersed; generally both liquids are dispersed, and the effect of the emulsifying agent is seen mainly in the subsequent coagulation process ; droplets of one liquid are prevented from coalescence while the others are not. In both dispersion and coagulation processes, the mechanicel treatment and the relative volumes, viscosities, densities and boundary tensions of the liquids are important factors, but their precise interrelation cannot be specified. Taylor ${ }^{1}$ recorded photographically the elongation of a single drop of liquid subjected to viscous drag in a second liquid, finding the effect of the viscosity of the drop relative to that of the external liquid to be striking, and some of his observations were afterwards given a mathematical basis by 'Timotika ${ }^{2}$. From Clay's ${ }^{a}$ experiments it appears likely that the bursting of droplets in turbulent flow takes place by a mechanism similar to that in the jet of an oil atomizer. The chances that a drop will burst decrease rapidly with the radius of the droplet below a certain value. 
Regarding the stability of emulsions, there are three ways in which the system can become less homogeneous : (i) by 'creaming', that is, by concentration of globules of the disperse phase towards the top or bottom of the emulsion; (ii) by clumping of individual globules without coalescence ; (iii) by coalescence of globules to form larger drops and eventually bulk liquid. Only the third represents instability in the sense of a reversal of the emulsification process ; but creaming may be objectionable and is usually minimized by fine dispersion and the addition of thickening agents to the external phase. Such thickening agents are generally materials which form viscous or thixotropic sols with the dispersion medium. There seems no doubt that the viscosity of the emulsion as a whole is closely related to the ease of emulsification, and the implication is that in the most stable emulsions the globules have a sufficiently low residual interfacial energy against the external phase to be substantially independent of one another. From the practical point of view, an emulsion with some tendency to thixotropy will be preferable to one with the minimum resistance to flocculation, since the latter will cream more readily. With certain emulsifying agents the stability of the emulsion obtained is dependent on the relative volumes of the phases, the concentration of agent and the mechanical treatment of the emulsion. Such systems are prone to show phase-type inversions or multiple emulsions, with abrupt changes in viscosity.

Most of the published work on the viscosity of emulsions has been carried out in connexion with the testing of equations relating the viscosity of an emulsion to that of the dispersion medium. Of such equations may be mentioned those of Taylor", Leviton and Leighton ${ }^{5}$, Richardson ${ }^{6}$, Broughton and Squires 7 and Hatschek ${ }^{8}$. A comprehensive series of experiments to test Hatschek's conclusions has been made by Sibree", who finds poor agreement between observed and calculated values, although an improvement in the equation may be effected by the introduction of a 'volume factor'. This is confirmed by Gabriel ${ }^{10}$. Richardson ${ }^{6}$ has developed an equation connecting apparent viscosity at an arbitrary rate of shear with the volume concentration. The work of Broughton and Squires ${ }^{7}$ has given a clear demonstration of the importance of the emulsifying agent in determining the viscosity of the emulsion. The works of Toms ${ }^{11}$, Lyttleton and Traxler ${ }^{12}$ and Eilers ${ }^{13}$ is also of interest in this connexion. No quantitative theory appears to have been advanced to account for the observed dependence of the viscosity of emulsions on the mean globule-size or the size distribution; homogenization of an emulsion increases its viscosity, sometimes considerably.

Our knowledge of this subject is still sketchy, Dr. Sumner concluded. In the past there has been a tendency to treat the flow of emulsions as an operation in solid geometry and mechanics; but it is now clear that the rheological behaviour is intimately connected with the nature of the interfacial film or phase, and the influence of globule-size distribution is more important than has hitherto been supposed.

In the ensuing discussion, Dr. A. H. Nissan mentioned that the constant of $2 \cdot 5$ in all the equations referred to is simply the value for the most probable range of globule-sizes. The size-range is important, and for uniform globules the constant may be as much as 4:3. Dr. Scott Blair remarked that Anstein and Reiner had shown that the Einstein law, including the $\mathbf{2 \cdot 5}$, holds for cement mortars of viscosity about
1017. Mr. A. de Waele directed attention to the fact that the equations of Richardson and of Broughton and Squires are approximations for the case when the viscosity ratio is nearly unity; some workers have lost sight of this and used them when the viscosity ratio is ten or more.

\section{Milk as an Emulsion}

Dr. R. Aschaffenburg continued with an account of "Milk as an Emulsion". Milk consists of an aqueous phase containing salts, lactose and colloids (mainly proteins) into which is dispersed fat. Though perhaps the most perfect single food, milk is not perfect as an emulsion because of its tendency to 'cream', which, however, may be overcome by homogenization. The slow break-up of this emulsion is possibly an aid to digestion. The size of the fat. globules varies from $0 \cdot 1 \mu$ to $10 \mu$; the size distribution depends upon several factors. The number of globules is very large : the weekly three-pint nonpriority milk ration in Britain contains about five thousand billion globules. Apart from creaming, the emulsion is stable, and it is not possible to extract the fat directly with solvents. About 1840 Ascherson suggested that this was due to a 'membrane' around the fat globules, and subsequent work, especially by L. S. Palmer, has confirmed this view. Electron microscope pictures of the fat globules, indeed, show each one surrounded by a dark layer and a hazy region, but the interpretation of such pictures is still the subject of much controversy.

The rising of cream is predominantly an effect of gravity, but, on account of clustering of the globules, the rate of rise is greater than would be expected from Stokes's law. Clustering is due to the presence, in milk, of an 'agglutinin' which, like bacterial agglutinins, is of a globulin nature.

Butter is now prepared by three main processes : (i) the classical method of churning, (ii) by the preparation of cream of a much higher fat percentage (about 80 per cent), followed by cooling and meohanical working, (iii) by the preparation of the 80 per cent fat cream, its conversion to pure butter oil and subsequent emulsification with the right amount of water, whey or skim milk. The classical method is a kind of flotation process with foam enrichment; it works best when the fat is neither completely 'solid' nor completely 'liquid', but it is not known why this should be so. Milk has a minimum foam stability at $20-30^{\circ}$, which has been shown to be due to the fat. Butter is not a straightforward oil-water emulsion, and its rheological properties vary with the method of manufacture. These properties offer an interesting field for further research.

\section{Formation and Flow of Emulsions}

Dr. E. G. Richardson, in a paper on "The Formation and Flow of Emulsions", said that there are two basic processes of emulsion manufacture. Either one liquid is squirted into another, or the two liquids are allowed to mix in turbulent motion. The first is the problem of the break-up of a liquid under the combined actions of the interfacial tension and the relative viscosity. In the main there are three types of such disintegration. In the first type, the jet becomes varicose. Rayleigh ${ }^{14}$ has examined the conditions for this to take place, and Tyler and Richardson ${ }^{15}$ have taken photographs of this type of break-up from capillary jets. In the second type, the jet becomes sinuous, and the resistance to the passage of the 'humps' becomes more important than the 
surface tension. This type has been dealt with mathematically by Weber ${ }^{18}$, and both Tyler ${ }^{17}$ and Ohmesorge ${ }^{18}$ have given criteria for the critical velocities for the change from the varicose to the sinuous forms. At high velocities in excess of the critical value, break-up is controlled by viscous and inertial forces. At low speeds, drops are formed when the varicosities acquire an amplitude sufficient to nick off the jet, and so tend to be of uniform size; whereas at high speed the drops are formed as 'lumps' sheared off the periphery and tend to show a greater dispersion of size.

Dr. Richardson showed photographs of jets and the results of drop-size measurements for jets of aniline, paraffin and oil, and benzene into water for different nozzle sizes and velocities. When jets issue from large nozzles at low speeds, the drops tend to oscillate about the spherical shape and eventually to burst into fragments. Such effects of deformation and circulation have been investigated by Bond and Newton ${ }^{2}$, and Taylor 4 has obtained an expression for the upper limit to the size of drop which can subsist while the fluid is being sheared. It is also possible to influence drop-size by acoustic agitation, and it appears that ultrasonic agitation can be used to produce greater fineness and uniformity of dispersion.

Regarding the flow of an emulsion, once formed, the viscosity is affected by (i) concentration of dispersed phase, (ii) drop-size distribution, (iii) rate of shear. In most practical emulsions the drops are so small that they act as rigid spheres when the emulsion is sheared. Experiments with benzene and water show that the viscosity increases rapidly with the concentration of the disperse phase, such that

$$
\log \eta / \eta_{0}=x_{c},
$$

where $\eta$ and $\eta_{0}$ are the viscosities of emulsion and 'solvent', $c$ is the concentration and $\chi$ is a sort of 'interphasal' compressibility. Broughton and Squires ${ }^{7}$ write

$$
\log \eta / \eta_{0}=\chi\left(c+c_{0}\right)
$$

where $c_{0}$ is an intercept on the axis.

The variations of viscosity can be given a partial explanation by supposing the process of shearing the emulsion to cause one row of globules to pass over another row. Slow motion will cause an upper row to pass over a lower row through a series of 'humps'. At higher rates of shear, the upper globules are supposed to 'jump' over the lower depressions without falling right in, and the viscous traction concerned in lifting them out of the 'hole' is less. Unpublished experiments by Iskander and Richardson on the effect of globule-size on viscosity show that the limiting viscosity at the highest rates of shear is roughly inversely proportional to the mean globulesize.

In the discussion on this paper, Mr. A. K. Soper took exception to the Broughton and Squires equation involving an intercept $c_{0}$ on the axis; Dr. A. S. C. Lawrence said that there was some evidence for the existence of an elastic skin around the globules. Mr. de Waele said that in solid-liquid systems there appears to be such a barrier, for the difference between the theoretical packing limit of 74 per cent for uniform spheres and the actual practical limit may be considerable. With a certain carbon black $(0.03 \mu-0.20 \mu)$ it is impossible to get more than 20 per cent into oil, and the product is not thixotropic -it fractures like a solid. With $\frac{1}{2}-\mu$ particles one gets nearer the theoretical 74 per cent limit, and then only does the phenomenon of dilatancy arise. The existence of a barrier layer would increase the stability of an emulsion.

Mr. J. E. Adamson remarked that globule-size distribution should be specified more precisely : in the simplest case of a Gaussian population, two factors are required (mean and standard deviation), and with more complicated distributions the moments about the mean must be considered. Dr. A. H. Nissan said that the flow of water through glass filters at various temperatures cannot be accounted for by viscosity effects alone, but one can get similar effects by adding materials which change the surface tension. This may be due to the formation of a surface layer on the glass which interferes with the normal mechanism of flow. Dr. W.H. Banks denied this, and said that it is very difficult in such experiments to be sure that the glass is not swollen; the electroviscous effect must be taken into account.

\section{G. W. HaRrison}

1 Taylor, Proc. Roy. Soc., A, 146, 501 (1934).

${ }^{2}$ Timotika, Proc. Roy. Soc., A, 150, 322 (1935); 153, 302 (1936).

'Clay, Proc. Roy. Acad. Sci. Amsterdam, 43, 852, 979 (1940).

- Taylor, Proc. Roy Soc., A, 138, 41 (1932).

${ }^{5}$ Leviton and Leighton, J. Phys. Chem., 40, 71 (1936).

- Richardson, Kolloid-Z., 65, 32 (1933).

${ }^{7}$ Broughton and Squires, J. Phys. Chem., 42, 253 (1938).

- Hatschek, Kolloid-Z., 7, 301 (1910) ; 8, 34 (1911) ; 11, 284 (1912); $12,238(1913)$

- Sibree, Trans. Farad. Soc., 26, 26 (1930); 27, 161 (1931).

10 Gabriel, "Technical Aspects of Emulsions" (London, 1935).

${ }^{11}$ Toms, J. Chem. Soc., 542 (1941).

${ }^{12}$ Lyttleton and Traxler, Indust. Eng. Chem., 40, 2115 (1948).

${ }^{13}$ Eilers, Kolloid-Z., 97, 313 (1941).

14 Rayleigh, Proc. Lond. Math. Soc., 10, 4 (1879).

${ }^{18}$ Tyler and Richardson, Proc. Phys. Soc., 37, 297 (1925).

${ }^{16}$ Weber, $Z$. angew. Math. Mech., 2, 136 (1932).

${ }^{17}$ Tyler, Phil. Mag., 16, 504 (1933).

18 Ohmesorge, $Z$. angew. Math. Mech., 16, 355 (1936).

10 Bond and Newton, Phil. Mag., 5, 794 (1928).

\section{GENETICS AT THE UNIVERSITY OF GLASGOW}

WITH the recent opening of new premises for the Department of Genetics, the development of teaching and research in genetics at the University of Glasgow has gone a step further. Of the two floors of the building, the top one is for research and can accommodate up to ten research workers in small laboratories ; the ground floor is reserved for undergraduate teaching and has laboratory accommodation and services for up to fifteen students.

- The research laboratories are suitable for any kind of genetical research with plants or animals, though the equipment has been chosen mainly in view of expanding the work with micro-organisms, the field of genetical research which has been in the forefront in Glasgow since 1943. The long-term line of research in the Department is an approach to the spatialtemporal organisation of biochemical processes in the cell, using the biochemical genetics of micro-organisms as a tool. Of the innumerable problems arising from this approach, those under actual investigation at present include the formal genetics of Aspergillus nidulans (a first systematic attempt to work with a homothallic species); the cytogenetics of a mycetozoon; the study of nuclear and cytoplasmic control of 'adaptive' enzyme systems; and the biochemical genetics of those biosyntheses which involve steps of millimicromolar order. Some of these problems have obvious implications for applied fields, for example, 